\title{
CITOLOGIA NO DIAGNÓSTICO INTRA-OPERATÓRIO DE NEOPLASIAS INTRACRANIANAS
}

\author{
L. C. Mattosinho-França *
}

Rolando A. Tenuto **

J. Guidugli NetTo ***

O conhecimento da natureza histológica das neoplasias intra-cranianas é de grande importância para o estabelecimento da conduta neuro-cirúrgica. Para os demais setores da patologia humana tem sido empregado com sucesso o diagnóstico histopatológico intra-operatório por cortes de congelação. Em neuropatologia, dadas as condições de pequena consistência dos fragmentos de tecido tumoral, friabilidade e escassa quantidade das amostras, os exames por cortes de congelação são de difícil emprêgo, sendo mais eficientes nas neoplasias de maior consistência, como os tumores meníngeos e de raizes nervosas. As neoplasias malígnas do sistema nervoso e, particularmente, as neoplasias gliais indiferenciadas, são as que maiores dificuldades apresentam ao exame por cortes de congelação sendo, ao mesmo tempo, os tumores de cujo diagnóstico preciso tem necessidade o neuro-cirurgião para o estabelecimento da conduta operatória. Na falta desse tipo de diagnóstico intra-operatório, a conduta cirúrgica é baseada antes nos dados clínicos, topográficos e radiológicos do que no conhecimento do tipo tumoral pròpriamente dito.

Para contornar tais dificuldades técnicas, já de algum tempo são conhecidos métodos para o estudo citológico intra-operatório de tumores cerebrais. As técnicas propostas variam tanto quanto à fixação, como quanto à coloração. Eisenhardt e Cushing ${ }^{5}$ e Deery ${ }^{2}$ empregam técnicas de coloração supra-vital, os primeiros usando vermelho-neutro, o segundo azure A e Eire Garnet B. Muitos fixam o espécime em líquido de Schaudinn, como Dudgeon e Patrick (cit. ${ }^{5}$ ) corando ulteriormente com hematoxilina e eosina. Russel, Krayembühl e Cairns ${ }^{25}$, e Figar 7 , usando o mesmo fixador, coram ulteriormente com azul de toluidina. Morris ${ }^{17}$ preconiza a fixação do esfregaço pelo calor e coloração com azul de metileno. Assim o fazem Liu e Adamkiewicz $^{14}$, Muratorio ${ }^{18}$ e Grattarola e Schiaffer ${ }^{8}$, enquanto Jane e Bertrand ${ }^{9}$, após fixação pelo calor coram com eosina e azul de metileno. Roca de Viñals ${ }^{23}, 24$ e Pickren e Burke ${ }^{21}$ usam o método de Papanicolaou, após fixação em álcool-éter. Cabieses-Molina ${ }^{1}$ fixa o esfregaço em formol a $10 \% \mathrm{im}$ -

* Chefe do Serviço de Anatomia Patológica, Hospital do Servidor Público do Estado de São Paulo; ** Chefe do Serviço de Neurocirurgia, Faculdade de Medicina; *** Médico do Serviço de Anatomia Patológica, Hospital do Servidor Público do Estado de São Paulo. 
pregnando-o, depois, com carbonato de prata amoniacal. Larson e Peterger ${ }^{11}$ e Papo e Colombo ${ }^{20}$ fixam os esfregaços em álcool-éter. Enquanto os primeiros coram com azul de metileno policrômico de Terry, os segundos usam simplesmente o azul de metileno. Ferrari ${ }^{6}$, sem fixação prévia, usa o microscópico de contraste de fase.

O objetivo deste trabalho é a apresentação dos resultados iniciais obtidos no estudo citológico intra-operatório de espécimens de tumores cerebrais, segundo técnica preconizada pelos autores.

\section{MATERIAL E METODOS}

Foram estudadas 7 neoplasias intra-cranianas. Após localização clínica e angiográfica das neoplasias, os pacientes foram submetidos à craniotomia. A técnica cirúrgica não será relatada, bem como os dados clínicos e de evolução, por fugirem ao objetivo desta apresentação. Nos tumores hemisféricos, foi inicialmente praticađa perfuração única da tábua óssea; nos demais foi feita craniotomia ampla. Após abertura da dura-mater, foram colhidos fragmentos de tecido tumoral com agulha de punçāo biópsia (Menghini). Os fragmentos obtidos foram distribuidos sob a forma de esfregaços em lâminas de microscopia, sem fixação prévia, por atrito entre duas lâminas. Parte dos esfregaços foi corada de imediato, sem fixação, em solução alcoólica de tionina a $5 \%$, e parte foi fixada em álcool-éter, para ulterior coloração pela técnica de Papanicolaou. Fragmentos remanescentes foram fixados em formalina, incluidos em parafina, e corados pela hematoxilina-eosina, sendo usados somo controles. O diagnóstico imediato foi dado com base nos achados dos esfregaços não fixados, corados pela tionina. Verificação dêsses resultados foi feita com base nos esfregaços fixados, corados pela técnica de Papanicolaou, e com o estudo histológico dos fragmentos de biópsia, incluidos em parafina.

\section{RESULTADOS}

Os resultados obtidos são apresentados de forma descritiva, segundo os casos individuais.

CASo 1 - (S63-669-) sexo masculino, branco, 51 anos de idade, tumor cerebral no hemisfério direito. Citologia - Os esfregaços são formados por células isoladas e agrupadas, tendo morfologia característica de astrocitos. Tais células têm citoplasma abundante e disposto em tôda a volta do núcleo. Tôdas as células têm prolongamentos múltiplos, sendo por vêzes um deles mais espesso que os demais. Os prolongamentos se estendem até grande distância do corpo celular e mostram pequenas ramificações. O citoplasma das células tem tonalidade ligeiramente acidófila. Os núcleos têm forma em geral ovóide, variando moderadamente em diâmetro. A cromatina é moderadamente granulosa. Algumas células são fusiformes, tendo núcleo polar e grosso prolongamento em uma das extremidades, assumindo aspecto de astroblastos. Algumas mitoses estão presentes. Raras células com prolongamentos bipolares, com caracteres de espongioblastos, são observadas. Diagnóstico: astrocitoma maligno. Biópsia - Fragmentos medindo até $2 \mathrm{~cm}$ de comprimento, tendo diâmetro de até $0,2 \mathrm{~cm}$. Tecido neoplásico formado por células em arranjo frouxo, tendo caracteres de astrocitos. Tais células têm citoplasma disposto sob a forma de prolongamentos múltiplos, ligeiramente acidófilos. Os núcleos variam bastante em forma e tamanho e têm cromatina grosseiramente granulosa. Há moderado hipercromatismo. Algumas células têm prolongamentos polares grosseiros. Pequeno número de mitoses está presente. Não há atipias vasculares. Diagnóstico: astrocitoma maligno. 
CASo 2 - (S63-2339), sexo masculino, branco, 72 anos de idade, tumor cerebral no hemisfério esquerdo. Citologia - Material constituído por células isoladas e agrupadas e por fragmentos de tecido. Há grande número de núcleos isolados, os quais têm moderado hipercromatismo, e grande variação no tamanho. Êsses núcleos tém nucléolos bastante evidentes. De maneira isolada e junto a fragmentos teciduais. nota-se a presença de células tumorais com citoplasma conservado. Tais células têm citoplasma abundante, acidofflico $e$, freqüentemente, distribuido apenas ao longo de polos opostos, formando prolongamentos longos. Os núcleos são volumosos, variando em forma e tamanho. Nucléolos evidentes estão presentes. A cromatina é grosseiramente granulosa. Alguns corpúsculos granulo-gordurosos estão presentes (fig. 1). Diagnóstico: glioblastoma multiforme. Biópsia - Material constando de fragmentos cilíndricos que medem até $1 \mathrm{~cm}$ de comprimento e $0,2 \mathrm{~cm}$ de diâmetro. Tecido neoplásico de aspecto polimorfo, formado por células em arranjo frouxo. As células tumorais mostram grande variação em forma e tamanho, havendo núcleos bastante volumosos. Os núcleos são hipercromáticos. Pequeno número de mitoses está presente. Alguns vasos têm endotélio estratificado. Focos de infiltrado linfocitário estão presentes ao redor de alguns vasos. Ãreas de necrose estāo presentes. Diagnóstico: glioblastoma multiforme.

CASO 3 - M.C., (S63-901), sexo feminino, branca, 38 anos de idade, tumor temporal esquerdo. Citologia - Material constituído por células neoplásicas isoladas e agrupadas e por grandes fragmentos de tecido. As células tumorais têm citoplasma ligeiramente acidófilo. A forma das células corresponde a astroblastos, espongioblastos e astrocitos atípicos. Muitas células mostram orangeofilia citoplasmática. Os núcleos têm tamanhos variados e cromatina grosseiramente granulosa. Nucléolos evidentes estão presentes. Alguns espongioblastos de grandes dimensões são observados, sendo predominantemente de forma bipolar. Mitoses estão presentes. Diagnóstico: glioblastoma multiforme. Biópsia - Material de biópsia incisional. Tecido neoplásico formado por células volumosas, dispostas em arranjo compacto. As células tumorais têm citoplasma abundante e freqüentemente de distribuição polar. Os núcleos variam em forma, tamanho e número. Os núcleos são de maneira geral volumosos e têm cromatina grosseiramente granulosa. Um ou mais nucléolos estão presentes em cada núcleo. o citoplasma é bastante acidófilo e há grande número de células com caracteres de astroblastos em arranjo peri-vascular. Outras células com caracteres espongioblásticos estão presentes, particularmente ao redor das áreas de necrose. Vasos com estratificação endotelial são observados. Diagnóstico: glioblastoma multiforme.

Caso 4 - M. F. S. (S63-738), sexo masculino, branco, 10 anos de idade, tumor de 3.0 ventrículo. Citologia - Material constituido predominantemente por fragmentos de tecido, os quais reproduzem a arquitetura do epitélio epidermóide. Não há atipias nucleares. Alguns iragmentos menores mostram arranjo concêntrico, com delimitação de pequenas pérolas córneas. Areas calcificadas amorfas estão presentes (fíg. 2). Diagnóstico: compatível com craniofaringeoma. Biópsia - fragmentos de biópsia incisional medindo até $1 \mathrm{~cm}$ na maior dimensão. Tecido neoplásico constituído por tecido conjuntivo frouxo, circundando cavidades irregulares revestidas por epitélio estratificado de tipo epidermóide. Este mostra camadas basal e malpighiana conspicuas, havendo na camada superficlal células arredondadas, queratinizadas, de permeio com material calcificado. Diagnóstico: craniofaringeoma.

Caso 5 - O.S., (S63-686), sexo masculino, branco, 8 anos de idade, tumor do vermis cerebelar, Citologia - Material constituido por células neoplásicas pequenas, de maneira geral isoladas. o citoplasma é pouco abundante e ligeiramente basofilico. Algumas células têm finos prolongamentos polares curtos. Os núcleos variam em forma e tamanho, sendo hipercromáticos. Algumas mitoses são obsercadas (fig. 3). Diagnóstico: Compativel com meduloblastoma. Biópsia - Material de biópsia incisional. Fragmentos maiores medindo $1,5 \mathrm{~cm}$ na maior dimensāo. Tecido neoplásico formado por células pequenas, em arranjo compacto, intercalado com extensas áreas de necrose. Células tumorais com citoplasma bastante escasso, não havendo neurópilo intersticial. Os núcleos variam de forma e tamanho, sen- 
do intensamente hipercromáticos. Muitos núcleos têm nucléolos evidentes. Moderado número de mitoses está presente. A vascularizaçāo capilar é escassa. Diagnóstico: Meduloblastoma.

Caso 6 - V.M., (S63-750), sexo masculino, branco, 61 anos de idade, tumor do hemisfério cerebelar esquerdo. Citologia - Material constituido por células isoladas e agrupadas. Há pequena quantidade de material acidofílico, formando um fundo no preparado. Há numerosos núcleos isolados. As células neoplásicas têm forma alongada, tendo algumas delas prolongamentos polares espessos. Algumas células são bastante volumosas, tendo abundante citoplasma acidofilico. Os núcleos variam em tamanho, tendo alguns deles vacúolo claro em seu interior. Muitas células têm núcleos múltiplos. Algumas mitoses estão presentes (fig. 4). Diagnóstico: variam em tamanho, tendo alguns deles vacúolo claro em seu interior. Muitas céplásico formando por células em arranjo frouxo, tendo grande polimorfismo. Predominam células alongadas, de tipo espongioblástico, havendo, entretanto, grande número de células com prolongamentos polares grosseiros, de típo astroblástico. Há extensas áreas de necrose. Os núcleos variam em forma e tamanho, sendo hipercromáticos. Há moderado número de mitoses. Vasos com endotélio atípico estão presentes. Diagnóstico: glioblastoma multiforme.

Caso 7 - S.A., (S64-213), sexo masculino, branco, 4 anos de idade, tumor de hemisfério cerebral direito. Citologia - Material constituido por células isoladas e agrupadas. As células têm citoplasma pouco abundante e mostram freqüentemente forma piramidal. O citoplasma é ligeiramente basofilico. Algumas células têm arranjo em corôa. Os núcleos săo pequenos, vesiculosos, tendo cromatina finamente granulosa e uniformemente distribuida. Diagnóstico: Compativel com ependimona. Biópsia - Fragmento de biópsia incisional, medindo até $1,5 \mathrm{~cm}$ na maior dimensão. O tecido neoplásico é formado por células pequenas, em arranjo compacto, sendo separadas entre sí por abundante rêde capilar. As células neoplásicas têm citoplasma pouco abundante e ligeiramente acidofilico. Os núcleos variam em forma e tamanho, sendo em geral pequenos. A cromatina nuclear é grosseiramente granulosa e uniformemente distribuída. Há numerosas mitoses. Diagnóstico: Ependimona.

Os resultados mostram que houve concordância absoluta dos achados citológicos com o resultado histológico em quatro casos; nos demais, os achados citológicos foram compativeis com o resultado histológico.

\section{DISCUSSAO E CONCLUSOES}

A finalidade precipua dos exames citológicos é a identificação da existência de neoplasia, sendo a classificação das neoplasias um objetivo antes da histologia do que da citologia 10,19. Entretanto, muitos autores usam o método citológico como instrumento para a classificação de neoplasias ${ }^{15}, 16,26$.

Os resultados apresentados são concordes com os obtidos com outras técnicas, já relatadas na literatura. Muratorio ${ }^{18}$, estudando 100 casos pela técnica de Morris, obteve $81 \%$ de acêrto diagnóstico em tumores meníngeos e $89 \%$ de acêrto em tumores neuro-ectodérmicos. Pickren e Burke ${ }^{21}$, estudando citològicamente 1819 tumores, dos quais 14 cerebrais, não citam erros diagnósticos. Larson e Peterger ${ }^{11}$ referem usar exclusivamente técnicas citológicas para o diagnóstico intra-operatório em neuropatologia.

Alguns autores salientam aspectos desfavoráveis das técnicas citológicas em neuropatologia. Jane e Bertrand ${ }^{9}$ citam como desvantagens a destruição da arquitetura, espessura irregular dos esfregaços, e difícil aplicação em 


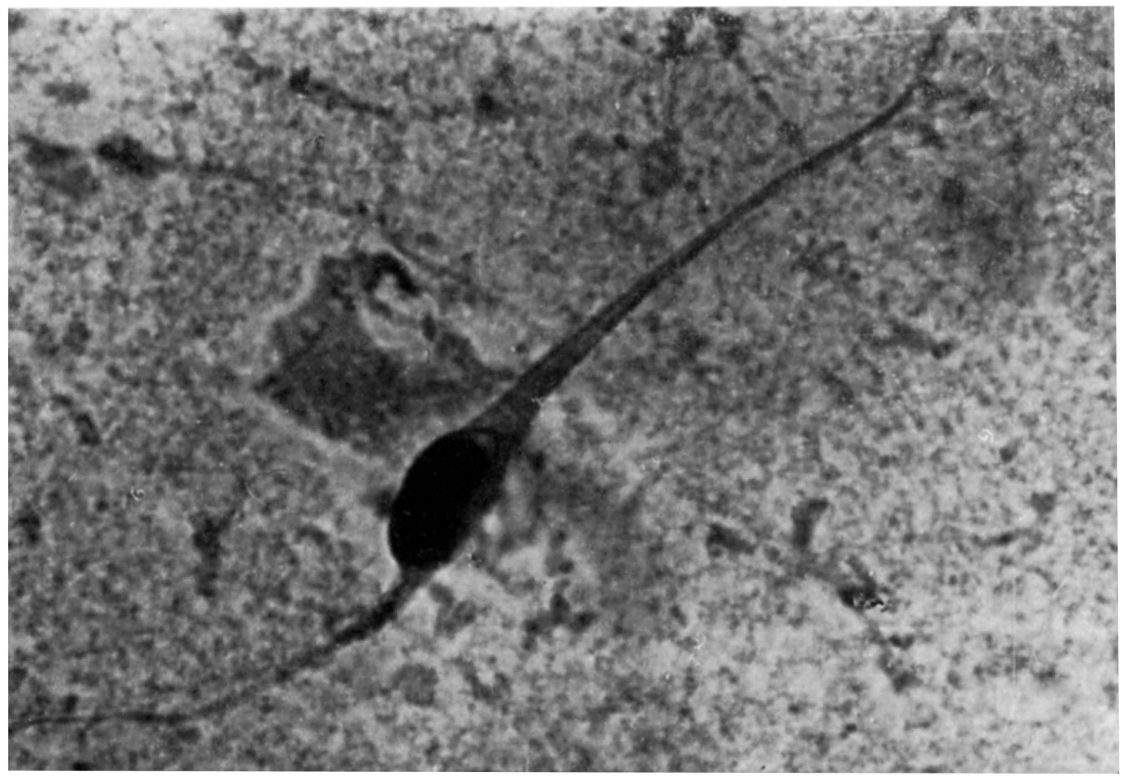

Fig. 1 - Caso 2. Citologia (1020x): espongioblasto, glioblastoma multiforme.

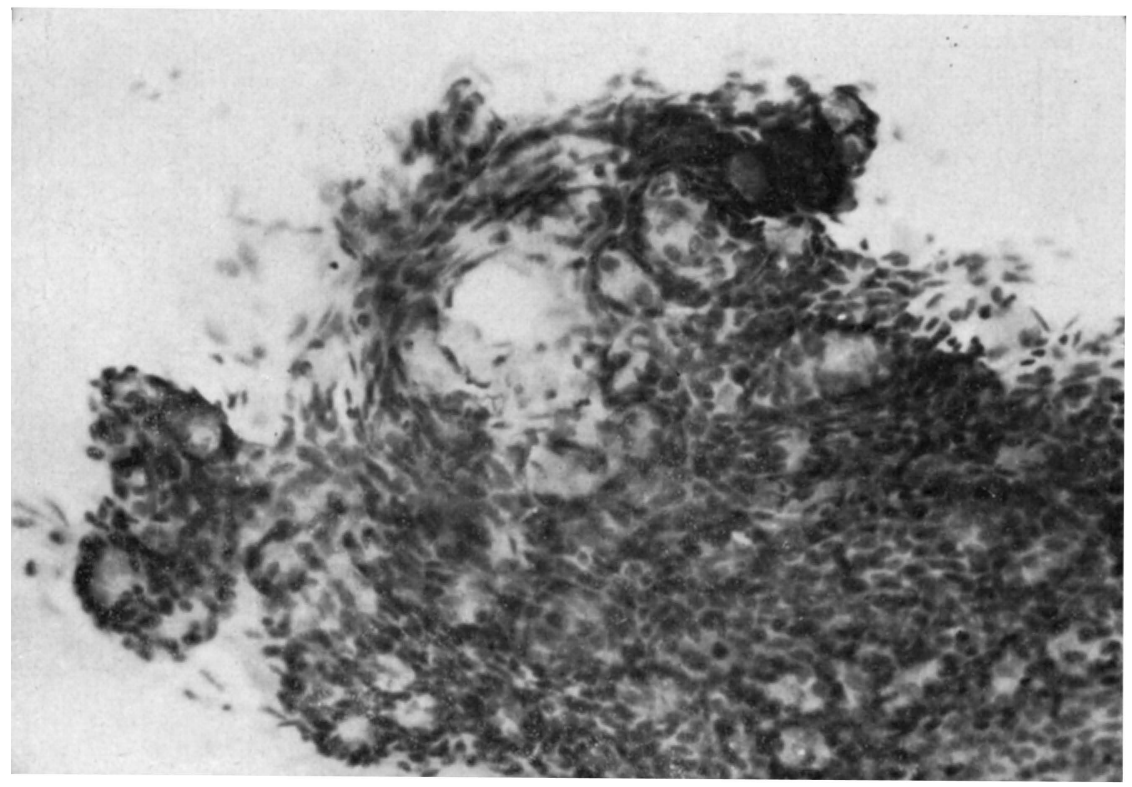

Fig. 2 - Caso 4. Citologia (310x): células de tipo malpighiano em craniofaringeoma. 


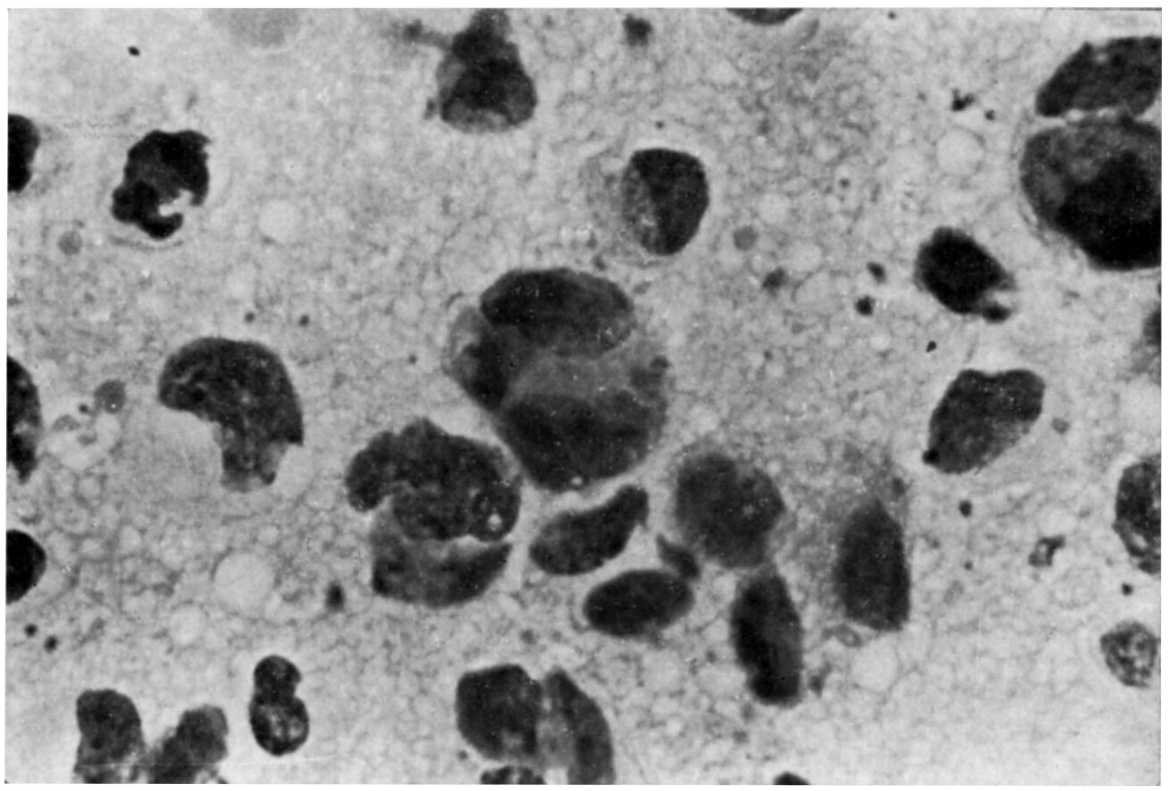

Fig. 3 - Caso 5. Citologia (1020x): meduloblastoma.

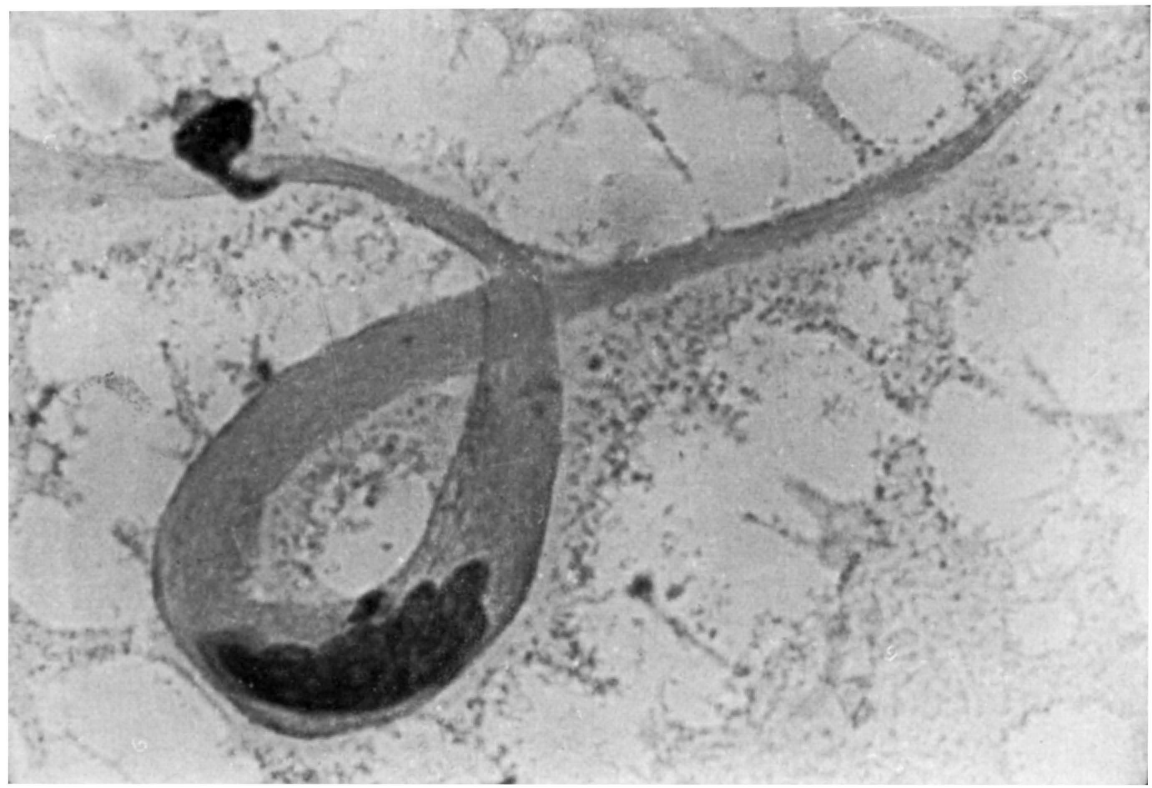

Fig. 4 - Caso 6. Citologia (1020x): espongioblasto atipico, glioblastoma multiforme. 
tumores de grande consistência. Liu e Adamkiewicz ${ }^{14}$ por motivos semelhantes, preferem a realização de diagnósticos intra-operatórios com cortes de congelação. A maior consistência de alguns tumores, entretanto, não impede a preparação de esfregaços para exame citológico, visto que Degtyareva e Burdenko ${ }^{3}$ e Degtyareva ${ }^{4}$ relatam casos de meningeomas e astrocitomas diagnosticados citològicamente.

A técnica aqui apresentada atende às demandas de rapidez e precisão de detalhes necessárias para o diagnóstico intra-operatório. Entretanto, não sendo as lâminas permanentes, visto serem montadas em água, esfregaços adicionais são fixados em álcool-éter e corados pela técnica de Papanicolaou, para fins de documentação e comparação com cortes histológicos da neoplasia em estudo. A interpretação dos resultados mostra dois aspectos distintos. De uma parte, a identificação da existência de neoplasia segue os cânones diagnósticos da citologia, podendo ser perfeitamente realizada por patologistas não experientes em neuropatologia. De outra parte, a identificação do tipo de neoplasia depende do grau de conhecimentos de citologia do sistema nervoso central, podendo assim a qualidade do diagnóstico variar de acôrdo com a experiência neuropatológica do observador. Ao nosso vêr a técnica apresentada substitue com vantagens a realização de cortes de congelação para o diagnóstico intra-operatório de tumores do sistema nervoso central.

\section{R E S U M O}

Os autores apresentam técnica de diagnóstico citológico para neoplasias do sistema nervoso central, aplicável ao diaznóstico rápido intra-operatório. A técnica é baseada em esfregaços não fixados, corados pela tionina. $O$ contrôle dos resultados foi feito tanto com esfregaços fixados e corados pela técnica de Papanicolaou, como mediante cortes histológicos do mesmo material. Os resultados obtidos são concordes com dados já publicados na literatura por outros autores. Os autores julgam que as técnicas citológicas suplantam com vantagens as técnicas histológicas de congelação, para o diagnóstico neuropatológico intra-operatório.

\section{S U M M A R Y}

\section{Cytology as an aid to operative diagnosis of intra-cranial tumors}

A technique based on unfixed thionine stained smears for cytologic diagnosis of intra-cranial tumors during neurosurgical operations is presented. The controls were both cytological (fixed, Papanicolaou stain) and histological (paraffin embedded). The technique substitutes with advantages the routine frozen section diagnosis. The results are in agreement with previous data reported in the literature using other cytologic techniques for the same purpose. 


\section{REFERENCIAS}

1. CABIESES-MOLINA, F. -- Rapid method for study of biopsy: acelerated method of Rio Hortega silver impregnation and its aplications to smears. Rev. Neuropsiquiat. (Lima) 13:651, 1950.

2. DEERY, E. M. - The neuro-surgical laboratory. Bull. Neurol. Inst. N.Y. 5: 127, 1936.

3. DEGTYAREVA, S. M. \& BURDENKO, S. - Cytological examination of meningeoma on contact smear preparations. Vop. Neirokhir 5:36, 1965 . Apud Exc. Med. VIII-A n.o 5464, 1966.

4. DEGTyareva, S. M. - Cytological picture of macroglial tumors. Arkh. Patol. 27:37, 1965. Apud. Exc. Med. VIII-A n.o 6265, 1966.

5. EISENHARDT, L. \& CUSHING, H. - Diagnosis of intracranial tumors by supra-vital technique. Am. J. Path. 6:541, 1930.

6. FERRARI, G. - Aspetti della struttura microscopica dell'encefalo rilevabili con la tecnica dell'esame citologico. G. Veneto Sci. Med. 17:403, 1962.

7. FIGAR, S. - Rapid diagnosis of brain tumors with the help of smears preparations. Casop Lek. Cesk. 91:873-879, 1952. Apud Exc. Med. VIII n.0 3947, 1953.

8. GRATTAROLA, F. R. \& SCHIFFER, D. - Tumori dell'encefalo in preparati estemporanei. Cancro 8:29; 1955. Apud Exc. Med. VIII n.o 4211, 1956.

9. JANE, J. A. \& BERTRAND, G. - A cytological method for the diagnosis os tumors affecting the C.N.S. J. Neuropath. Exp. Neurol. 21:400, 1962.

10. KOSS, L. G. \& DURFEE, G. R. - Diagnostic Cytology and its Histopathologic Basis. J. B. Lippincott \& Co, Philadelphia, 1961.

11. LARSON, C. P. \& PETERGER, C. C. - Smear method for rapid diagnosis of neuro-surgical material. Am. J. Clin. Path. 22:916, 1952.

12. LARSON, C. P. - Rapid diagnosis on neuro-surgical material. Northwest Medical $49: 869,1950$.

13. LE BEAU, J.; NGO-NGOC, N. C. \& NUOVO, V. - Le cytodiagnostic dans les tumeurs maligns du cerveau. Sem. Hop. Paris 42:215, 1966.

14. LIU, C. T. \& ADAMKIEWICZ, L. L. - Presumptive diagnosis of brain tumor by the rapid smear technique. Arch. Neurol. Psychiat. (Chicago), 64:149, 1950.

15. MATTOSINHO-FRANÇA, L. C.; TAVARES DE LIMA, M.; LEMOS, S. \& VELLONI, M. - Observações sôbre a correlação entre os diagnósticos citológicos e histológicos em neoplasias pulmonares. Rev. Assoc. med. bras. 12:335, 1966.

16. MATtósinho-FRANÇA, L. C. \& SPINA-FRANÇA, A. - Método de Papanicolaou e pesquisa de células neoplásicas no líquido céfalorraqueano. Rev. paulista Med. 67:203, 1965.

17. MORRIS, A. A. - The use of the smear technique in the rapid histological diagnosis of tumors of the central nervous system: description of a new staining method. J. Neurosurg. 4:497, 1947. 
18. MURATORIO, A. - Contributo alla conoscenza dei metodi estemporanei per la diagnosi intra-operatoria dei tumori endocranici: il metodo de Morris. Boll. Oncol. (Roma) 29 (supl. 1) : 546, 1955.

19. PAPANICOLAOU, G. M. - Atlas of Exfoliative Cytology. Harvard University Press, Cambridge, Massachusetts, 1954.

20. PAPO, I. \& COLOMBO, F. - Possibilities and limitations of the extemporaneous examination by imprint and smears in the per-operative diagnosis of intra-cranial tumors. Minerva Neurochir. 3:134, 1959.

21. PICKREN, J. \& BURKE, E. - Adjuvant cytology to frozem sections. Acta Cytologica $7: 164,1963$.

22. ROBSON, J. T. - The value of rapid method tissue diagnosis to the neurosurgeon. Northwest Medical 49:872, 1950.

23. ROCA DE VINALS, R. - Aportaciones al diagnóstico citológico de los tumores cerebrales. Rev. Esp. Oncol. 4:113, 1955.

24. ROCA DE VINALS, R. - Aportaciones al diagnóstico citológico de los tumores cerebrales Folia Clin. Int. (Barcelona) 6:297, 1956.

25. RUSSEL, D. S.; KRAYEMBUHL, H. \& CAIRNS, H. - The wet-film technique in the histological diagnosis of intra-cranial tumors: a rapid method. J. Path. Bact. 45:501, 1937.

26. UMIKER, W. - Typing of eronchogenic carcinoma. AMA Arch. Path. 71:295, 1961.

Serviço de Anatomia Patológica - Hospital do Servidor Público Estadual Rua Pedro de Toledo 1800 - São Paulo, SP - Brasil. 